\title{
Type I Total Anomalous Pulmonary Venous Return
}

National Cancer Institute

\section{Source}

National Cancer Institute. Type I Total Anomalous Pulmonary Venous Return. NCI

Thesaurus. Code C98590.

Total anomalous pulmonary venous return in which the pulmonary veins drain into the right atrium via the superior vena cava. 